\title{
A Description of the RC2(r) Encryption Algorithm
}

Status of this Memo

This memo provides information for the Internet community. It does not specify an Internet standard of any kind. Distribution of this memo is unlimited.

Copyright Notice

Copyright (C) The Internet Society (1998). All Rights Reserved.

1. Introduction

This memo is an RSA Laboratories Technical Note. It is meant for informational use by the Internet community.

This memo describes a conventional (secret-key) block encryption algorithm, called RC2, which may be considered as a proposal for a DES replacement. The input and output block sizes are 64 bits each. The key size is variable, from one byte up to 128 bytes, although the current implementation uses eight bytes.

The algorithm is designed to be easy to implement on 16-bit microprocessors. On an IBM AT, the encryption runs about twice as fast as DES (assuming that key expansion has been done).

\subsection{Algorithm description}

We use the term "word" to denote a 16-bit quantity. The symbol + will denote twos-complement addition. The symbol \& will denote the bitwise "and" operation. The term XOR will denote the bitwise "exclusive-or" operation. The symbol will denote bitwise complement. The symbol will denote the exponentiation operation. The term MOD will denote the modulo operation.

There are three separate algorithms involved:

Key expansion. This takes a (variable-length) input key and produces an expanded key consisting of 64 words K[0],..., K[63]. 
Encryption. This takes a 64-bit input quantity stored in words $R[0], \ldots, R[3]$ and encrypts it "in place" (the result is left in $R[0], \ldots, R[3])$.

Decryption. The inverse operation to encryption.

2. Key expansion

Since we will be dealing with eight-bit byte operations as well as 16-bit word operations, we will use two alternative notations

for referring to the key buffer:

For word operations, we will refer to the positions of the buffer as K[0], ..., K[63]; each K[i] is a 16-bit word.

For byte operations, we will refer to the key buffer as L[0], ..., L[127]; each L[i] is an eight-bit byte.

These are alternative views of the same data buffer. At all times it will be true that

$$
K[i]=L[2 * i]+256 * L[2 * i+1]
$$

(Note that the low-order byte of each $\mathrm{K}$ word is given before the high-order byte.)

We will assume that exactly $\mathrm{T}$ bytes of key are supplied, for some $\mathrm{T}$ in the range $1<=\mathrm{T}<=128$. (Our current implementation uses $\mathrm{T}=8$.) However, regardless of $\mathrm{T}$, the algorithm has a maximum effective key length in bits, denoted $\mathrm{T} 1$. That is, the search space is $2^{\wedge}\left(8^{\star} \mathrm{T}\right)$, or $2^{\wedge} \mathrm{T} 1$, whichever is smaller.

The purpose of the key-expansion algorithm is to modify the key buffer so that each bit of the expanded key depends in a complicated way on every bit of the supplied input key.

The key expansion algorithm begins by placing the supplied T-byte key into bytes L[0], ..., L[T-1] of the key buffer.

The key expansion algorithm then computes the effective key length in bytes T8 and a mask TM based on the effective key length in bits T1. It uses the following operations:

$\mathrm{T} 8=(\mathrm{T} 1+7) / 8 ;$

$\mathrm{TM}=255 \mathrm{MOD} 2^{\wedge}(8+\mathrm{T} 1-8 * \mathrm{~T} 8) ;$

Thus TM has its $8-(8 * \mathrm{~T} 8-\mathrm{T} 1)$ least significant bits set. 
For example, with an effective key length of 64 bits, $\mathrm{T} 1=64, \mathrm{~T} 8=8$ and $\mathrm{TM}=$ oxff. With an effective key length of 63 bits, $\mathrm{T} 1=63, \mathrm{~T} 8$ $=8$ and $\mathrm{TM}=0 \times 7 \mathrm{f}$.

Here PITABLE[0], ..., PITABLE[255] is an array of "random" bytes based on the digits of PI = 3.14159... . More precisely, the array PITABLE is a random permutation of the values $0, \ldots, 255$. Here is the PITABLE in hexadecimal notation:

\begin{tabular}{|c|c|c|c|c|c|c|c|c|c|c|c|c|c|c|c|}
\hline 0 & 1 & 2 & 3 & 4 & 5 & 0 & 1 & 0 & & a & b & G & $d$ & & f \\
\hline : $:$ d9 & 78 & f9 & C 4 & 19 & $d d$ & b5 & ed & 28 & e 9 & $\mathrm{fd}$ & 79 & $4 a$ & $\mathrm{a} 0$ & d8 & \\
\hline : $c 6$ & $7 e$ & 37 & 83 & $2 \mathrm{~b}$ & 76 & 53 & $8 e$ & 62 & $4 \mathrm{C}$ & 64 & 88 & 44 & $8 b$ & $\mathrm{fb}$ & \\
\hline 17 & $9 a$ & 59 & 5 & 87 & o3 & $4 \mathrm{f}$ & 13 & 61 & 45 & $6 d$ & $8 d$ & 09 & 1 & d & \\
\hline $\mathrm{bd}$ & $8 f$ & 40 & eb & 86 & b 7 & $7 \mathrm{~b}$ & $\mathrm{Ob}$ & $\mathrm{fo}$ & 95 & 21 & 22 & $5 c$ & $6 \mathrm{~b}$ & $4 e$ & \\
\hline 54 & $d 6$ & 65 & 93 & $\mathrm{ce}$ & 60 & $\mathrm{~b} 2$ & $1 \mathrm{C}$ & 73 & 56 & $\mathrm{CO}$ & 14 & a 7 & $8 c$ & $\mathrm{f} 1$ & \\
\hline 12 & 75 & $\mathrm{Ca}$ & $1 \mathrm{f}$ & $3 b$ & be & e4 & $\mathrm{d} 1$ & 42 & $3 d$ & $\mathrm{~d} 4$ & 30 & a 3 & $3 c$ & $\mathrm{~b} 6$ & \\
\hline $6 f$ & $\mathrm{bf}$ & $0 e$ & da & 46 & 69 & 07 & 57 & 27 & $\mathrm{f} 2$ & $1 d$ & $9 b$ & $\mathrm{bc}$ & 4 & 43 & \\
\hline f 8 & 11 & C 7 & $\mathrm{f} 6$ & 90 & ef & $3 e$ & e 7 & 06 & c3 & d5 & $2 f$ & c8 & 66 & $1 e$ & \\
\hline 08 & e8 & ea & de & 80 & 52 & ee & f 7 & 84 & aa & 72 & ac & 35 & $4 d$ & $6 a$ & \\
\hline 96 & $1 a$ & $\mathrm{~d} 2$ & 71 & $5 a$ & 15 & 49 & 74 & $4 b$ & $9 f$ & 0 & be & 04 & -8 & 4 & \\
\hline c2 & e0 & 41 & $6 e$ & $0 f$ & 51 & $\mathrm{cb}$ & $\mathrm{CC}$ & 24 & 91 & $a f$ & 50 & a1 & $\mathrm{f} 4$ & 70 & \\
\hline 99 & $7 c$ & $3 a$ & 85 & 23 & $\mathrm{~b} 8$ & $\mathrm{~b} 4$ & $7 a$ & $\mathrm{fC}_{\mathrm{C}}$ & 02 & 36 & $5 b$ & 25 & 55 & 97 & \\
\hline $2 d$ & $5 d$ & fa & 98 & e3 & $8 a$ & 92 & $\mathrm{ae}$ & 05 & $d f$ & 29 & 10 & 67 & $6 c$ & $\mathrm{ba}$ & c \\
\hline d3 & 00 & e 6 & $c f$ & e1 & $9 e$ & a 8 & 20 & 63 & 16 & 01 & $3 f$ & 58 & e2 & 89 & \\
\hline $0 \mathrm{~d}$ & 38 & 34 & 11 & $a b$ & 33 & ff & bo 0 & $\mathrm{bb}$ & 48 & $\mathrm{OC}_{\mathrm{C}}$ & $5 f$ & b 9 & b1 & $\mathrm{cd}$ & \\
\hline c5 & f3 & $\mathrm{db}$ & 47 & e 5 & a 5 & $9 c$ & 77 & $0 \mathrm{a}$ & a 6 & 20 & 68 & $\mathrm{fe}$ & $7 \mathrm{f}$ & $\mathrm{C} 1$ & \\
\hline
\end{tabular}

The key expansion operation consists of the following two loops and intermediate step:

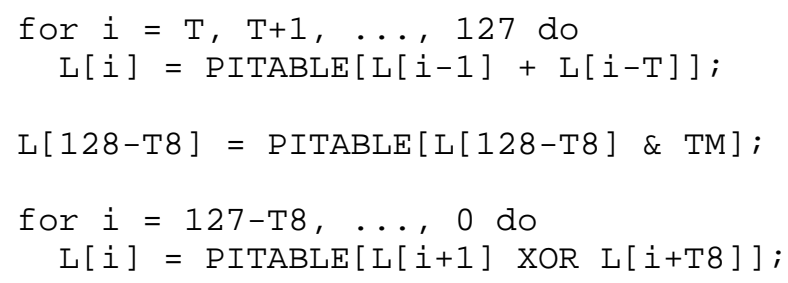

(In the first loop, the addition of L[i-1] and L[i-T] is performed modulo 256.)

The "effective key" consists of the values L[128-T8],..., L[127]. The intermediate step's bitwise "and" operation reduces the search space for L[128-T8] so that the effective number of key bits is T1. The expanded key depends only on the effective key bits, regardless 
of the supplied key $K$. Since the expanded key is not itself modified during encryption or decryption, as a pragmatic matter one can expand the key just once when encrypting or decrypting a large block of data.

3. Encryption algorithm

The encryption operation is defined in terms of primitive "mix" and "mash" operations.

Here the expression "x rol k" denotes the 16-bit word $\mathrm{x}$ rotated left by $\mathrm{k}$ bits, with the bits shifted out the top end entering the bottom end.

3. 1 Mix up R[i]

The primitive "Mix up R[i]" operation is defined as follows, where $\mathrm{s}[0]$ is 1, s[1] is 2, s[2] is 3, and s[3] is 5, and where the indices of the array $R$ are always to be considered "modulo 4," so that R[i-1] refers to $R[3]$ if $i$ is 0 (these values are

"wrapped around" so that $R$ always has a subscript in the range 0 to 3 inclusive) :

$R[i]=R[i]+K[j]+(R[i-1] \& R[i-2])+((\sim R[i-1]) \& R[i-3])$;

$j=j+1 ;$

$R[i]=R[i] \operatorname{rol} s[i]$;

In words: The next key word $K[j]$ is added to $R[i]$, and $j$ is advanced. Then R[i-1] is used to create a "composite" word which is added to R[i]. The composite word is identical with R[i-2] in those positions where $R[i-1]$ is one, and identical to $R[i-3]$ in those positions where $\mathrm{R}[i-1]$ is zero. Then $\mathrm{R}[\mathrm{i}]$ is rotated left by $\mathrm{s}[\mathrm{i}]$ bits (bits rotated out the left end of $R[i]$ are brought back in at the right). Here $j$ is a "global" variable so that $\mathrm{K}[\mathrm{j}]$ is always the first key word in the expanded key which has not yet been used in a "mix" operation.

3.2 Mixing round

A "mixing round" consists of the following operations:

Mix up R[0]

Mix up R[1]

Mix up R[2]

Mix up R[3] 


\subsection{Mash $R[i]$}

The primitive "Mash R[i]" operation is defined as follows (using the previous conventions regarding subscripts for $R$ ):

$R[i]=R[i]+K[R[i-1] \& 63]$;

In words: R[i] is "mashed" by adding to it one of the words of the expanded key. The key word to be used is determined by looking at the low-order six bits of R[i-1], and using that as an index into the key array $\mathrm{K}$.

3.4 Mashing round

A "mashing round" consists of:

Mash R[0]

Mash $\mathrm{R}[1]$

Mash $R[2]$

Mash R[3]

\subsection{Encryption operation}

The entire encryption operation can now be described as follows. Here $j$ is a global integer variable which is affected by the mixing operations.

1. Initialize words $R[0], \ldots, R[3]$ to contain the 64-bit input value.

2. Expand the key, so that words K[0], ..., K[63] become defined.

3. Initialize j to zero.

4. Perform five mixing rounds.

5. Perform one mashing round.

6. Perform six mixing rounds.

7. Perform one mashing round.

8. Perform five mixing rounds.

Note that each mixing round uses four key words, and that there are 16 mixing rounds altogether, so that each key word is used exactly 
once in a mixing round. The mashing rounds will refer to up to eight of the key words in a data-dependent manner. (There may be repetitions, and the actual set of words referred to will vary from encryption to encryption.)

4. Decryption algorithm

The decryption operation is defined in terms of primitive operations that undo the "mix" and "mash" operations of the encryption algorithm. They are named "r-mix" and "r-mash" ( $r$ - denotes the reverse operation).

Here the expression "x ror k" denotes the 16-bit word $\mathrm{x}$ rotated right by $\mathrm{k}$ bits, with the bits shifted out the bottom end entering the top end.

\subsection{R-Mix up R[i]}

The primitive "R-Mix up R[i]" operation is defined as follows, where $\mathrm{s}[0]$ is $1, \mathrm{~s}[1]$ is 2, $\mathrm{s}[2]$ is 3, and $\mathrm{s}[3]$ is 5, and where the indices of the array $R$ are always to be considered "modulo 4," so that R[i-1] refers to $R[3]$ if $i$ is 0 (these values are "wrapped around" so that $R$ always has a subscript in the range 0 to 3 inclusive):

$R[i]=R[i] \operatorname{ror} s[i] ;$

$R[i]=R[i]-K[j]-(R[i-1] \& R[i-2])-((\sim R[i-1]) \& R[i-3])$; $j=j-1$;

In words: $\mathrm{R}[\mathrm{i}]$ is rotated right by $\mathrm{s}$ [i] bits (bits rotated out the right end of $\mathrm{R}[\mathrm{i}]$ are brought back in at the left). Here j is a "global" variable so that $\mathrm{K}[\mathrm{j}]$ is always the key word with greatest index in the expanded key which has not yet been used in a "r-mix" operation. The key word $\mathrm{K}[\mathrm{j}]$ is subtracted from $\mathrm{R}[\mathrm{i}]$, and $j$ is decremented. $R[i-1]$ is used to create a "composite" word which is subtracted from R[i]. The composite word is identical with R[i-2] in those positions where $\mathrm{R}[\mathrm{i}-1]$ is one, and identical to $\mathrm{R}[\mathrm{i}-3]$ in those positions where $\mathrm{R}[\mathrm{i}-1]$ is zero.

4.2 R-Mixing round

An "r-mixing round" consists of the following operations:

$\mathrm{R}-\mathrm{Mix}$ up $\mathrm{R}[3]$

$\mathrm{R}-\mathrm{Mix}$ up $\mathrm{R}[2]$

$\mathrm{R}-\mathrm{Mix}$ up $\mathrm{R}[1]$

R-Mix up R[0] 


\subsection{R-Mash R[i]}

The primitive "R-Mash R[i]" operation is defined as follows (using the previous conventions regarding subscripts for R):

$R[i]=R[i]-K[R[i-1] \& 63]$;

In words: R[i] is "r-mashed" by subtracting from it one of the words of the expanded key. The key word to be used is determined by looking at the low-order six bits of R[i-1], and using that as an index into the key array $\mathrm{K}$.

4.4 R-Mashing round

An "r-mashing round" consists of:

R-Mash R[3]

$\mathrm{R}-\mathrm{Mash} \mathrm{R}[2]$

$\mathrm{R}-\mathrm{Mash} \mathrm{R}[1]$

$\mathrm{R}-\mathrm{Mash} \mathrm{R}[\mathrm{O}]$

\subsection{Decryption operation}

The entire decryption operation can now be described as follows. Here $j$ is a global integer variable which is affected by the mixing operations.

1. Initialize words R[0], ..., R[3] to contain the 64-bit ciphertext value.

2. Expand the key, so that words $\mathrm{K}[0], \ldots, \mathrm{K}[63]$ become defined.

3. Initialize j to 63 .

4. Perform five r-mixing rounds.

5. Perform one r-mashing round.

6. Perform six r-mixing rounds.

7. Perform one r-mashing round.

8. Perform five r-mixing rounds.

5. Test vectors

Test vectors for encryption with RC2 are provided below. 
All quantities are given in hexadecimal notation.

Key length (bytes) $=8$

Effective key length (bits) $=63$

Key $=0000000000000000$

Plaintext $=0000000000000000$

Ciphertext $=$ ebb773f9 93278eff

Key length (bytes) $=8$

Effective key length (bits) $=64$

Key $=$ ffffffff ffffffff

Plaintext $=$ ffffffff ffffffff

Ciphertext $=278 \mathrm{~b} 27 e 4$ 2e2f0d49

Key length (bytes) $=8$

Effective key length (bits) $=64$

Key $=3000000000000000$

Plaintext $=1000000000000001$

Ciphertext $=30649$ edf 9 be $7 \mathrm{~d} 2 \mathrm{c} 2$

Key length (bytes) $=1$

Effective key length (bits) $=64$

Key $=88$

Plaintext $=0000000000000000$

Ciphertext $=61 \mathrm{a} 8 \mathrm{a} 244$ adacccfo

Key length (bytes) $=7$

Effective key length (bits) $=64$

Key $=88 \mathrm{bca} 90 \mathrm{e} 90875 \mathrm{a}$

Plaintext $=0000000000000000$

Ciphertext $=6 \mathrm{ccf} 4308974 \mathrm{c} 267 \mathrm{f}$

Key length (bytes) $=16$

Effective key length (bits) = 64

Key $=88 \mathrm{bca} 90 \mathrm{e} 90875 \mathrm{a} f \mathrm{f}$ 0f79c384 627bafb2

Plaintext $=0000000000000000$

Ciphertext $=1 \mathrm{a} 807 \mathrm{~d} 272 \mathrm{bbe} 5 \mathrm{db} 1$

Key length (bytes) $=16$

Effective key length (bits) $=128$

Key $=88 \mathrm{bca} 90 \mathrm{e} 90875 \mathrm{a} 7 \mathrm{f}$ of79c384 627bafb2

Plaintext $=0000000000000000$

Ciphertext $=2269552 \mathrm{a}$ b0f85ca6

Key length (bytes) $=33$

Effective key length (bits) $=129$

Key $=88 \mathrm{bca90e} 90875 \mathrm{a} f \mathrm{f}$ of79c384 627bafb2 16f80a6f 85920584

c42fceb0 be255daf $1 e$ 
Plaintext $=0000000000000000$

Ciphertext $=5 \mathrm{~b} 78 \mathrm{~d} 3 \mathrm{a} 4$ 3dffflf 1

6. RC2 Algorithm Object Identifier

The Object Identifier for RC2 in cipher block chaining mode is IC2CBC OBJECT IDENTIFIER

1. If the number $\mathrm{EKB}$ of effective key bits is in the range 1-255, then the version number is given by Table[EKB], where the 256-byte translation table Table[] is specified below. Table[] specifies a permutation on the numbers 0-255; note that it is not the same table that appears in the key expansion phase of RC2.

2. If the number $\mathrm{EKB}$ of effective key bits is in the range 256-1024, then the version number is simply EKB.

The default number of effective key bits for RC2 is 32 . If RC2-CBC is being performed with 32 effective key bits, the parameters should be supplied as a simple IV, rather than as a SEQUENCE containing a version and an IV. 


\begin{tabular}{|c|c|c|c|c|c|c|c|c|c|c|c|c|c|c|c|c|}
\hline & 0 & 1 & 2 & 3 & 4 & 5 & 6 & 7 & 8 & 9 & 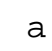 & $\mathrm{b}$ & . & d & e & \\
\hline 0 : & & & & & & & C & & 2 & & & 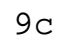 & & & & \\
\hline 0 & 30 & 04 & $\mathrm{~b} 6$ & d & & $d f$ & 2 & & f7 & b & 5 & $9 b$ & 1 & b & 21 & \\
\hline 0 : & 41 & $9 f$ & e1 & d 9 & $4 a$ & $4 d$ & $9 e$ & da & $\mathrm{a} 0$ & 68 & $2 \mathrm{C}$ & c3 & 27 & $5 f$ & 80 & \\
\hline 0 : & $3 e$ & ee & $\mathrm{fb}$ & 95 & $1 a$ & fe & $\mathrm{ce}$ & a 8 & 34 & a 9 & 13 & fo & a 6 & $3 f$ & 8 & \\
\hline $0:$ & 78 & 24 & af & 23 & 52 & C1 & 67 & 17 & f5 & 66 & 90 & e7 & e8 & 07 & 8 & \\
\hline 0 : & 48 & e 6 & $1 e$ & 53 & f3 & 92 & a 4 & 72 & $8 c$ & 08 & 15 & $6 e$ & 86 & 00 & 84 & \\
\hline $0:$ & $\mathrm{f} 4$ & $7 \mathrm{f}$ & $8 a$ & 42 & 19 & f6 & $\mathrm{db}$ & $\mathrm{cd}$ & 14 & $d$ & 50 & 12 & $\mathrm{ba}$ & $3 c$ & 06 & \\
\hline 0 : & ec & b3 & 35 & 11 & a 1 & 88 & $8 e$ & $2 \mathrm{~b}$ & 94 & 99 & b7 7 & 71 & 74 & d3 & e 4 & \\
\hline 0 & $3 a$ & de & 96 & $0 e$ & $\mathrm{bc}$ & $0 \mathrm{a}$ & ed & 77 & $\mathrm{fC}_{\mathrm{C}}$ & 7 & $6 \mathrm{~b}$ & 0 & 79 & 9 & 62 & \\
\hline $0:$ & $d 7$ & $\mathrm{CO}$ & $\mathrm{d} 2$ & $7 \mathrm{C}$ & $6 a$ & $8 b$ & 22 & a 3 & $5 b$ & 05 & $5 d$ & 02 & 75 & d5 & 61 & \\
\hline 0 : & 18 & $8 \mathrm{f}$ & 55 & 51 & $\mathrm{ad}$ & $1 \mathrm{f}$ & $0 \mathrm{~b}$ & $5 e$ & 85 & e 5 & c2 & 57 & 63 & $\mathrm{ca}$ & $3 d$ & \\
\hline 0 : & $\mathrm{b} 4$ & c5 & $\mathrm{CC}$ & 70 & $\mathrm{~b} 2$ & 91 & 59 & $0 \mathrm{~d}$ & 47 & 20 & C 8 & $4 \mathrm{f}$ & 58 & e0 & 01 & \\
\hline 0 : & 16 & 38 & C4 & $6 \mathrm{f}$ & $3 b$ & $0 \mathrm{f}$ & 65 & 46 & be & $7 e$ & $2 d$ & $7 \mathrm{~b}$ & 82 & f9 & 40 & \\
\hline 0 : & 10 & 73 & f8 & eb & 26 & C 7 & 87 & 97 & 25 & 54 & $\mathrm{~b} 1$ & 28 & aa & 98 & $9 d$ & \\
\hline $0:$ & 64 & $6 d$ & $7 a$ & $\mathrm{~d} 4$ & 10 & 81 & 44 & ef & 49 & d6 & 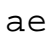 & $2 e$ & $\mathrm{dd}$ & 76 & $5 c$ & \\
\hline . & 7 & $1 \mathrm{C}$ & C9 & 09 & 69 & $9 a$ & 83 & $\mathrm{Cf}$ & 29 & 39 & 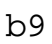 & e 9 & $4 \mathrm{C}$ & $f f$ & 43 & \\
\hline
\end{tabular}

A. Intellectual Property Notice

RC2 is a registered trademark of RSA Data Security, Inc. RSA's copyrighted RC2 software is available under license from RSA Data security, Inc.

B. Author's Address

Ron Rivest

RSA Laboratories

100 Marine Parkway, \#500

Redwood City, CA 94065 USA

Phone: (650) 595-7703

EMail: rsa-labsersa.com 
C. Full Copyright statement

Copyright (C) The Internet Society (1998). All Rights Reserved.

This document and translations of it may be copied and furnished to others, and derivative works that comment on or otherwise explain it or assist in its implementation may be prepared, copied, published and distributed, in whole or in part, without restriction of any kind, provided that the above copyright notice and this paragraph are included on all such copies and derivative works. However, this document itself may not be modified in any way, such as by removing the copyright notice or references to the Internet society or other Internet organizations, except as needed for the purpose of developing Internet standards in which case the procedures for copyrights defined in the Internet Standards process must be followed, or as required to translate it into languages other than English.

The limited permissions granted above are perpetual and will not be revoked by the Internet society or its successors or assigns.

This document and the information contained herein is provided on an "AS IS" basis and THE INTERNET SOCIETY AND THE INTERNET ENGINEERING TASK FORCE DISCLAIMS ALL WARRANTIES, EXPRESS OR IMPLIED, INCLUDING BUT NOT LIMITED TO ANY WARRANTY THAT THE USE OF THE INFORMATION HEREIN WILL NOT INFRINGE ANY RIGHTS OR ANY IMPLIED WARRANTIES OF MERCHANTABILITY OR FITNESS FOR A PARTICULAR PURPOSE. 